\title{
SU(5/3) Superalgebra and Its Representations of Fundamental Particles
}

\author{
Chang-Ho $\mathrm{Kim}^{a}$ and Seung Kook $\mathrm{Kim}^{b}$ \\ Department of Physics and Basic Science Research Institute, Seonam University, \\ Namwon, Jeonbuk 590-711, Korea \\ Young-Jai Park ${ }^{c}$ \\ Department of Physics, Sogang University, Seoul 121-742, Korea \\ and \\ Center for Quantum Spacetime, Sogang University, Seoul 121-742, Korea
}

\begin{abstract}
We study the lowest dimensional typical and atypical representations of $\mathrm{SU}(5 / 3)$ superalgebra as a possible unified gauge theory having a natural SU(5) subalgebra with $\mathrm{SU}(3)$ extra structure, which will be used to accommodate three generations of fundamental particles. By using Kac-Dynkin weight techniques, we find that all known quarks and leptons can be really accommodated in one atypical irreducible representation of $\mathrm{SU}(5 / 3)$.
\end{abstract}

PACS Nos: 04.65.+e, 11.30.Pb, 02.20.+b, 11.30.-j, 12.10.-g.

${ }^{a}$ E-mail address : chhjkim@gmail.com

${ }^{b}$ E-mail address : skandjh@empal.com

${ }^{c}$ E-mail address : yjpark@sogang.ac.kr 


\section{Introduction}

There is no doubt about the Standard Model [1,2] with gauge group (or Lie algebra) $\mathrm{SU}(3)_{\mathrm{c}} \otimes \mathrm{SU}(2)_{\mathrm{w}} \otimes \mathrm{U}(1)_{\mathrm{Y}}$. However, the theory contains many unexplained patterns and free parameters so one tries to find a simpler model by fitting the data of the Standard Model into a larger symmetry. The simplest model including one generation of fundamental particles is a grand unified theory(GUT) based upon the Lie algebra $\mathrm{SU}(5)$ which is proposed by Georgi and Glashow [3]. Even though the SU(5) GUT model shows many promising features, it unified the Standard Model only partly. For instance all known fundamental fermions cannot be contained in a single irreducible representation (irrep) of SU(5). Other models, for example $\mathrm{SO}(10)$ [4] or E6 [5], containing $\mathrm{SU}(5)$ as a subalgebra were proposed [6]. Such GUTs also had a problem in the proton decay that was predicted in the $\mathrm{SU}(5)$ model but not be confirmed by experiments.

From then particle physics has searched supersymmetric theories such as supergravities, superstrings, $M$ and $F$ theories. There have been many efforts to find suitable superalgebras which describe these supersymmetric theories [7, 8, 9]. Supersymmetric extensions of Poincaré algebra in arbitrary dimensional space-time were reviewed, and their representations(reps) for the supermultiplets of all known supergravity theories were extensively searched by Strathdee [10,11]. Moreover, $M$ and $F$ theories [12] have been also tackled from the point of view of the general properties of the superalgebra [13]. Kac proposed possible connections to the supersymmetric Standard Model with the reps of two exceptional superalgebras $\mathrm{E}(3 / 6)$ and $\mathrm{E}(3 / 8)$ that have the maximal compact subgroup $\mathrm{SU}(3) \otimes \mathrm{SU}(2) \otimes \mathrm{U}(1)$ [14]. Recently, Javis suggested polynomial deformations of Lie superalgebras and their reps, where the supermultiplets do not have any superpartners [15].

On the other hand, a new unification in terms of the Lie superalgebra SU(1/5) has been given [16, 17], and Stoilova and Jeugt showed some elegant features of the

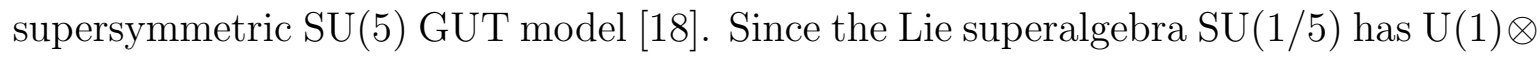
$\mathrm{SU}(3)_{\mathrm{c}} \otimes \mathrm{SU}(2)_{\mathrm{w}} \otimes \mathrm{U}(1)_{\mathrm{Y}}$ as a subalgebra, it essentially contains the Lie algebra of the Standard Model. All the fundamental fermions of a single generation (so all leptons 
and quarks of left and right chirality and their antiparticles) are inside one single irrep of $\mathrm{SU}(1 / 5)$. The first $\mathrm{U}(1)$ in the subalgebra level could be considered as a tool of distinguishing between the different generations with same irreps of $\mathrm{U}(1) \otimes \mathrm{SU}(3)_{\mathrm{c}} \otimes$ $\mathrm{SU}(2)_{\mathrm{w}} \otimes \mathrm{U}(1)_{\mathrm{Y}}$.

In this paper, we study typical and atypical representations of $\mathrm{SU}(5 / n)$ superalgebra as a possible unified gauge theory having a natural SU(5) subalgebra structure with $\mathrm{SU}(n)$ extra one, which will be used to accommodate of fundamental particles. We show that the three generations of quarks and leptons are in one atypical irrep of $\mathrm{SU}(5 / 3)$ superalgebra by using Kac-Dynkin weight techniques. In Sec. 2, the mathematical structure of the $\mathrm{SU}(5 / n)$ superalgebra is given. In Sec. 3, we briefly recapitulate the previous work [18] of the SU(5/1) unification in terms of Kac-Dynkin weight techniques. In Sec. 4, we investigate one irrep for the unification of three generations by using the $\mathrm{SU}(5 / 3)$ superalgebra, which will be shown the lowest one among $\mathrm{SU}(5 / n)$ superalgebras. Here, by analyzing a branching pattern $\mathrm{SU}(5 / 1) \otimes \mathrm{SU}(2) \subset \mathrm{SU}(5 / 3)$, we explicitly show that all known three generations of fundamental particles could be iden-

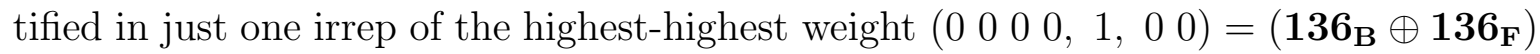
of $\mathrm{SU}(5 / 3)$. The last section contains conclusion.

\section{Mathematical Structures of SU(5/n) Superalge- bra}

The Kac-Dynkin diagram of $\mathrm{SU}(5 / n)$ Lie superalgebra is

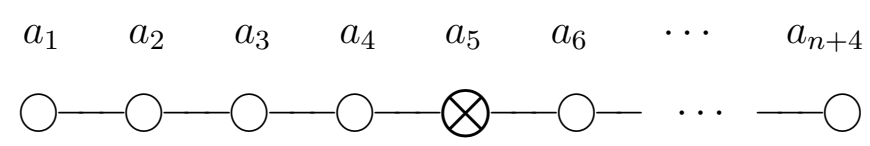

where the set $\left(\begin{array}{llll}a_{1} & a_{2} & \cdots & a_{n+4}\end{array}\right)$ determines the highest-highest weight vector of an irrep of $\mathrm{SU}(5 / n)[7,8,9,19]$. Each weight component $a_{i}$ for $i \neq 5$ of the highest-highest weight vector should be a nonnegative integer, while $a_{5}$ could be any complex number. The first four white nodes corresponding $\left(a_{1}, a_{2}, a_{3}, a_{4}\right)$ and the $n-1$ white nodes for 
$\left(a_{6}, \cdots, a_{n+4}\right)$ denote $\mathrm{SU}(5)$ and $\mathrm{SU}(n)$ bosonic subalgebra, respectively, and the fifth node for the component $a_{5}$ is responsible to supersymmetric generators. The graded Cartan matrix for $\mathrm{SU}(5 / n)$ is

$$
\left[\begin{array}{rrrrrrrrr}
2 & -1 & 0 & 0 & 0 & 0 & 0 & \cdots & 0 \\
-1 & 2 & -1 & 0 & 0 & 0 & 0 & \cdots & 0 \\
0 & -1 & 2 & -1 & 0 & 0 & 0 & \cdots & 0 \\
0 & 0 & -1 & 2 & -1 & 0 & 0 & \cdots & 0 \\
0 & 0 & 0 & -1 & 0 & 1 & 0 & \cdots & 0 \\
0 & 0 & 0 & 0 & -1 & 2 & -1 & \cdots & 0 \\
. & . & . & . & . & -1 & 2 & \cdots & 0 \\
. & . & . & . & . & . & . & \ddots & -1 \\
0 & 0 & 0 & 0 & 0 & \cdots & 0 & -1 & 2
\end{array}\right] .
$$

Note that each positive/negative simple even root $\alpha_{i}^{ \pm}(i=1, \cdots, n+4, i \neq 5)$ corresponds to the $i$-th column of the graded Cartan matrix, while the positive/negative simple odd root $\beta_{5}^{5 \pm}$ corresponds to the 5-th column of the matrix. Other odd roots are obtained by

$$
\beta_{5}^{i \pm}=\left[\alpha_{i}^{ \pm}, \beta_{5}^{(i+1) \pm}\right], \quad i=1,2,3,4,
$$

and

$$
\beta_{j+1}^{5 \pm}=\left[\beta_{j}^{5 \pm}, \alpha_{j}^{ \pm}\right], \quad j=5,6, \cdots, n+4 .
$$

In general, the irreps of $\mathrm{SU}(m / n)$ are divided into two types, which are typical and atypical $[7,9]$. All atypical irreps of $\mathrm{SU}(5 / n)$ are characterized by the fifth component $a_{5}$ of the highest-highest weight. The atypicality condition of $\mathrm{SU}(5 / n)$ is given by

$$
a_{5}=\sum_{k=6}^{j} a_{k}-\sum_{k=i}^{4} a_{k}-10+i+j, \quad 1 \leq i \leq 5,5 \leq j \leq n-1 .
$$

The typical irreps of $\mathrm{SU}(5 / n)$ consist of $5 n+1$ floors, and have equal bosonic and fermionic degrees of freedom. An atypical irrep is obtained by terminating some odd root strings in a full weight system when $a_{5}$ satisfies the relation in Eq. (5) for specific $i$ 's and $j$ 's. 
The lowest dimensional typical rep of $\mathrm{SU}(5 / n)$ is $\left(\begin{array}{llllll}0 & 0 & 0 & a_{5}, 0 & \cdots & \cdots\end{array}\right)=2_{B}^{5 n-1} \oplus 2_{F}^{5 n-1}$, for $a_{5} \neq n-1, \cdots, 2,1,0,-1,-2,-3,-4$, where the subscripts $B$ and $F$ denote bosons and fermions respectively, such as

$$
\begin{aligned}
& \mid \text { gnd }>\quad\left(\begin{array}{llllllll}
0 & 0 & 0 & 0 & a_{5}, & 0 & \cdots & 0
\end{array}\right) \\
& \downarrow \beta_{5}^{5-} \\
& \mid 1 \text { st }>\quad\left(\begin{array}{lllllllllll}
0 & 0 & 0 & 1, & a_{5}, & 1 & 0 & \cdots & 0
\end{array}\right) \\
& \swarrow \beta_{5}^{4-} \quad \searrow \beta_{6}^{5-} \\
& \text { 2nd }>\quad\left(\begin{array}{llllllllllll}
0 & 0 & 1 & 0, & a_{5}+1, & 2 & 0 & \cdots & 0
\end{array}\right) \quad\left(\begin{array}{lllllllll}
0 & 0 & 0 & 2, & a_{5}-1, & 0 & 1 & \cdots & 0
\end{array}\right) \\
& \swarrow \beta_{5}^{3-} \quad \searrow \beta_{6}^{5-} \quad \swarrow \beta_{5}^{4-} \quad \searrow \beta_{7}^{5-}
\end{aligned}
$$

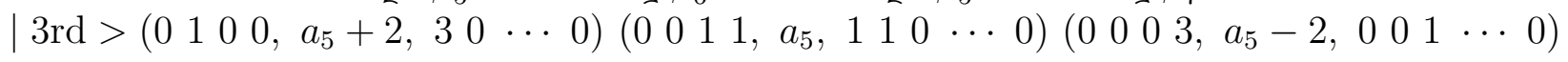

$$
\begin{aligned}
& \mid(5 n-1) \text {-th }>\quad\left(\begin{array}{lllllllll}
1 & 0 & 0 & 0 & a_{5}-n+5, & 0 & \cdots & 0 & 1
\end{array}\right) \\
& \mid 5 n \text {-th }>\quad\left(0000, a_{5}-n+5,0 \cdots 0\right)
\end{aligned}
$$

The even and odd floors alternate between fermions and bosons by the successive actions of odd roots.

If one take the value $a_{5}$ to satisfy the atypical condition, then the corresponding odd root strings are terminated from the typical irrep in Eq. (6), hence the the weight system ( $\left.\begin{array}{lllllll}0 & 0 & 0 & 0\end{array}, a_{5}, 0 \cdots 0\right)$ should be separated in several atypical irreps.

The fundamental rep of $\mathrm{SU}(5 / n)$ is $(10 \cdots 0)$ which has the substructure of $(\mathbf{5} \oplus \mathbf{n})$ in $\mathrm{SU}(5) \otimes \mathrm{SU}(n) \otimes \mathrm{U}(1)$ bosonic subalgebra basis as follows:

$$
\begin{aligned}
& \mid \text { gnd }>\left(\begin{array}{llllllll}
1 & 0 & 0 & 0,0, & 0 & 0 & \cdots & 0
\end{array}\right)=(\mathbf{5}, \mathbf{1})_{\mathbf{n}} \\
& \mid \text { 1st }>\quad\left(\begin{array}{lllllll}
0 & 0 & 0 & 0,1,1 & 0 & \cdots & 0
\end{array}\right)=(\mathbf{1}, \mathbf{n})_{\mathbf{5}}
\end{aligned}
$$

where the subscripts denote $\mathrm{U}(1)$ values corresponding the weight component $a_{5}$. The $\mathrm{U}(1)$ supercharge generator is $\operatorname{Diag}(n, n, n, n, n, \underbrace{5, \cdots, 5}_{n})$ to satisfy the supertraceless condition.

The complex conjugation of the fundamental rep of $\mathrm{SU}(5 / n)$ for $n \geq 2$ is $(0 \cdots 01)=$ $\left(\mathbf{n}^{*} \oplus \mathbf{5}^{*}\right)$ such as 


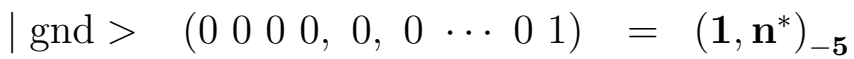

$$
\begin{aligned}
& \mid \text { 1st }>\quad\left(\begin{array}{llllllll}
0 & 0 & 0 & 1,-1, & 0 & \cdots & 0 & 0
\end{array}\right)=\left(\mathbf{5}^{*}, \mathbf{1}\right)_{-\mathbf{n}} \text {. }
\end{aligned}
$$

The Eq. (8) reduces to $\left(\begin{array}{lllll}0 & 0 & 0 & 0 & 0\end{array}-1\right)$ for the case $\mathrm{SU}(5 / 1)$ :

$$
\begin{aligned}
& \mid \text { gnd }>\quad\left(\begin{array}{llll}
0 & 0 & 0 & 0,-1
\end{array}\right)=\mathbf{1}_{-\mathbf{5}} \\
& \mid \text { 1st }>\quad\left(\begin{array}{llll}
0 & 0 & 0 & 1,-1
\end{array}\right)=\mathbf{5}_{{ }_{-} \mathbf{1}} .
\end{aligned}
$$

Like an usual algebra $\mathrm{SU}(5+n)$ the superalgebra $\mathrm{SU}(5 / n)$, where $n \neq 5$, has $(5+n)^{2}-1$ roots which consists of even roots for the subalgebras $\mathrm{SU}(5), \mathrm{SU}(n)$ and $\mathrm{U}(1)$ plus $10 n$ odd roots. The even and odd roots are in the adjoint rep $\left(\begin{array}{lllll}1 & 0 & \cdots & 0 & 1\end{array}\right)$ such as

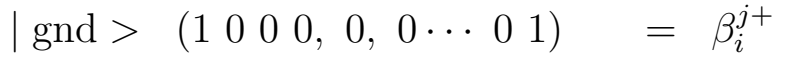

$$
\begin{aligned}
& \mid 1 \text { st }>\quad\left(\begin{array}{lllllll}
1 & 0 & 0 & 1,-1, & 0 & \cdots & 0
\end{array}\right)=\mathrm{SU}(5) \\
& \left(\begin{array}{llllllll}
0 & 0 & 0 & 0,0 & 0,0 & \cdots & 0
\end{array}\right) \quad=\quad \mathrm{U}(1) \\
& \left(\begin{array}{lllllllll}
0 & 0 & 0 & 0,1,1 & 0 & \cdots & 0 & 1
\end{array}\right)=\mathrm{SU}(n) \\
& \text { | 2nd }>\left(\begin{array}{llllllll}
0 & 0 & 0 & 1, & 1 & 1 & 0 & \cdots
\end{array}\right)=\beta_{i}^{j-} \text {. }
\end{aligned}
$$

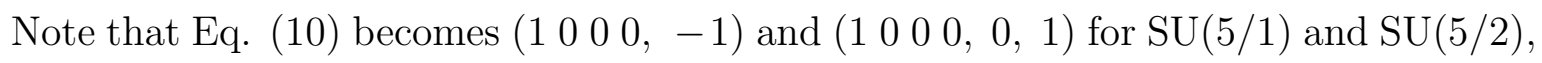
respectively as following

$$
\begin{aligned}
& \mid \text { gnd }>\left(\begin{array}{lllll}
1 & 0 & 0 & 0,-1
\end{array}\right)=\beta_{5}^{j+}
\end{aligned}
$$

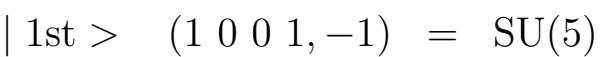

$$
\begin{aligned}
& \left(\begin{array}{lllll}
0 & 0 & 0 & 0,0
\end{array}\right)=\mathrm{U}(1) \\
& \text { | 2nd > }\left(\begin{array}{lllll}
0 & 0 & 0 & 1, & 0
\end{array}\right)=\beta_{5}^{j-} \text {. }
\end{aligned}
$$




$$
\begin{aligned}
& \mid \text { gnd }>\left(\begin{array}{llllll}
1 & 0 & 0 & 0 & 0,1
\end{array}\right)=\beta_{i}^{j+} \\
& \mid \text { 1st }>\quad\left(\begin{array}{llllll}
1 & 0 & 0 & 1,-1,0
\end{array}\right)=\mathrm{SU}(5) \\
& \left(\begin{array}{llllll}
0 & 0 & 0 & 0, & 0,0
\end{array}\right)=\mathrm{U}(1) \\
& \left(\begin{array}{lllll}
0 & 0 & 0 & 0,1,2
\end{array}\right)=\mathrm{SU}(2) \\
& \text { | 2nd > (l } \left.\begin{array}{lllll}
0 & 0 & 0 & 1,1,1
\end{array}\right)=\beta_{i}^{j-} \text {, }
\end{aligned}
$$

\section{Typical and Atypical Representations of SU(5/1)}

The lowest dimensional typical irrep of $\mathrm{SU}(5 / 1)$ is $\left(\begin{array}{l}0 \\ 0\end{array} 00, a_{5}\right)$ with $a_{5} \neq-4,-3,-2,-1,0$ such as

$$
\begin{aligned}
& \mid \text { gnd }>\quad\left(\begin{array}{lllll}
0 & 0 & 0 & 0 & a_{5}
\end{array}\right)=\mathbf{1} \\
& \downarrow \beta_{5}^{5-}
\end{aligned}
$$

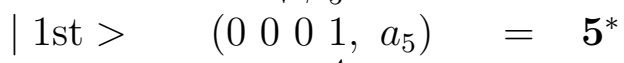

$$
\begin{aligned}
& \downarrow \beta_{5}^{4-} \\
& \text { | 2nd > (l } \left.\begin{array}{lllll}
0 & 1 & 0 & 0 & a_{5}+1
\end{array}\right)=10^{*} \\
& \downarrow \beta_{5}^{3-} \\
& \mid \text { 3rd }>\quad\left(\begin{array}{lllll}
0 & 1 & 0 & 0 & a_{5}+2
\end{array}\right)=\mathbf{1 0} \\
& \downarrow \beta_{5}^{2-} \\
& \mid \text { th }>\quad\left(\begin{array}{lllll}
1 & 0 & 0 & 0 & a_{5}+3
\end{array}\right)=\mathbf{5} \\
& \downarrow \beta_{5}^{1-} \\
& \mid 5 \text { th }>\left(\begin{array}{lllll}
0 & 0 & 0 & 0 & a_{5}+4
\end{array}\right)=\mathbf{1} .
\end{aligned}
$$

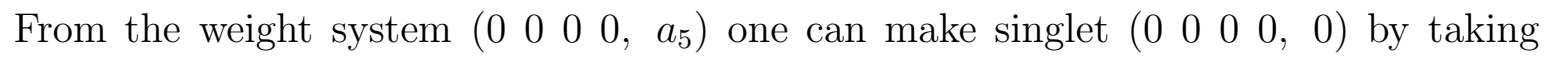
$a_{5}=0$. In this case a positive/negative odd root $\beta_{5}^{5 \pm}$ must be destroyed in Eq. (13). Notice that the other five floors make another atypical rep $\left(\begin{array}{llll}0 & 0 & 0 & 1,0\end{array}\right)$. Indeed, the Eq. (13) is separated into two independent atypical irreps by removing one of the positive/negative odd roots $\beta_{5}^{i \pm}$ 's by using the atypical condition in Eq. (5). We get two independent atypical reps, where the first one is starting from the ground floor and another one composed by remaining floors such as 


$$
\begin{aligned}
& a_{5}=0: \quad\left(\begin{array}{lllll}
0 & 0 & 0 & 0 & 0
\end{array}\right) \quad 0 \quad \oplus \quad\left(\begin{array}{lllll}
0 & 0 & 0 & 1, & 0
\end{array}\right) \\
& a_{5}=-1:\left(\begin{array}{lllll}
0 & 0 & 0 & 0,-1
\end{array}\right) \quad \oplus \quad\left(\begin{array}{lllll}
0 & 0 & 1 & 0, & 0
\end{array}\right) \\
& a_{5}=-2:\left(\begin{array}{lllll}
0 & 0 & 0 & 0,-2
\end{array}\right) \quad \oplus \quad\left(\begin{array}{lllll}
0 & 1 & 0 & 0, & 0
\end{array}\right) \\
& a_{5}=-3:\left(\begin{array}{lllll}
0 & 0 & 0 & 0,-3
\end{array}\right) \quad \oplus \quad\left(\begin{array}{lllll}
1 & 0 & 0 & 0, & 0
\end{array}\right) \\
& a_{5}=-4:\left(\begin{array}{lllll}
0 & 0 & 0 & 0,-4
\end{array}\right) \oplus \quad\left(\begin{array}{lllll}
0 & 0 & 0 & 0, & 0
\end{array}\right) .
\end{aligned}
$$

Recently, Stoilova and Jeugt had shown that the fundamental fermions fit inside just one irrep of $\mathrm{SU}(1 / 5)[18]$. Here we briefly reconstruct the theory in terms of $\mathrm{SU}(5 / 1)$ superalgebra.

The typical irrep $\left(\begin{array}{lllll}0 & 0 & 0 & 0 & a_{5}\end{array}\right)$ has substructures that are relevant to the supersym-

\begin{tabular}{|c|c|c|c|}
\hline & $\mathrm{SU}(5 / 1)$ & $\mathrm{SU}(5) \otimes \mathrm{U}(1)$ & $\mathrm{SU}_{c}(3) \otimes \mathrm{SU}_{w}(2) \otimes \mathrm{U}_{Y}(1)$ \\
\hline gnd > & $\left(\begin{array}{lllll}0 & 0 & 0 & 0, & a_{5}\end{array}\right)$ & $\left(\begin{array}{llll}0 & 0 & 0 & 0\end{array}\right)_{5 a_{5}}$ & $\left(\begin{array}{ll}0 & 0\end{array}\right)\left(\begin{array}{ll}0 & )_{0}\end{array}\right.$ \\
\hline 1 st $>$ & $\left(\begin{array}{lllll}0 & 0 & 0 & 1, & a_{5}\end{array}\right)$ & $\left(\begin{array}{llll}0 & 0 & 0 & 1\end{array}\right)_{5 a_{5}+4}$ & 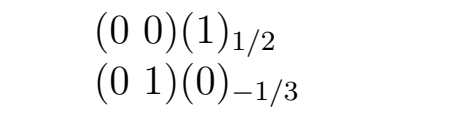 \\
\hline 2 nd $>$ & $\left(\begin{array}{lllll}0 & 0 & 1 & 0, & a_{5}+1\end{array}\right)$ & $\left(\begin{array}{llll}0 & 0 & 1 & 0\end{array}\right)_{5 a_{5}+8}$ & $\begin{array}{l}\left(\begin{array}{ll}0 & 0\end{array}\right)(0)_{1} \\
\left(\begin{array}{ll}0 & 1\end{array}\right)(1)_{1 / 6} \\
\left(\begin{array}{ll}1 & 0\end{array}\right)(0)_{-2 / 3}\end{array}$ \\
\hline $3 r d>$ & $\left(\begin{array}{lllll}0 & 1 & 0 & 0, & a_{5}+2\end{array}\right)$ & $\left(\begin{array}{llll}0 & 1 & 0 & 0\end{array}\right)_{5 a_{5}+12}$ & $\begin{array}{l}\left(\begin{array}{ll}0 & 1\end{array}\right)(0)_{2 / 3} \\
\left(\begin{array}{ll}1 & 0\end{array}\right)(1)_{-1 / 6} \\
\left(\begin{array}{ll}0 & 0\end{array}\right)(0)_{-1}\end{array}$ \\
\hline 4 th $>$ & $\left(\begin{array}{lllll}1 & 0 & 0 & 0, & a_{5}+3\end{array}\right)$ & $\left(\begin{array}{llll}1 & 0 & 0 & 0\end{array}\right)_{5 a_{5}+16}$ & $\begin{array}{l}\left(\begin{array}{ll}1 & 0\end{array}\right)(0)_{1 / 3} \\
\left(\begin{array}{ll}0 & 0)(1)_{-1 / 2}\end{array}\right.\end{array}$ \\
\hline 5 th $>$ & $\left(\begin{array}{lllll}0 & 0 & 0 & 0, & a_{5}+4\end{array}\right)$ & $\left(\begin{array}{llll}0 & 0 & 0 & 0\end{array}\right)_{5 a_{5}+20}$ & $\left(\begin{array}{ll}0 & 0\end{array}\right)(0)_{0}$ \\
\hline
\end{tabular}
metric extension of $\mathrm{SU}(5)$ model. Since the rep contains fermionic and bosonic parts simultaneously, the odd floors $\left[\mathbf{5}^{*} \oplus \mathbf{1 0} \oplus \mathbf{1}\right]$ contain the original fermions, while the even floors $\left[\mathbf{1} \oplus \mathbf{1 0}^{*} \oplus \mathbf{5}\right]$ could be identified with the superpartners.

We obtain the branching rule such as

Note that an atypical rep $\left(\begin{array}{llll}0 & 0 & 0 & 0,\end{array}-4\right)$ of SU(5/1) exactly accommodates well known one generation $\left[5^{*} \oplus \mathbf{1 0}\right]$ of $\mathrm{SU}(5)$ by decoupling the singlet fermion at the fifth floor in Eq. (13) which is included in the $\mathrm{SO}(10)$ model, while $(0001,0)$ which is complex 


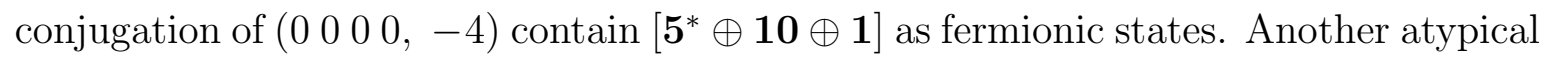
rep $\left(\begin{array}{llll}0 & 0 & 0 & 0,\end{array}-3\right)$ also contain $\left[5^{*} \oplus \mathbf{1 0}\right]$ but ends at the third floor.

We find an atypical rep $\left(\begin{array}{llllll}0 & 0 & 0 & 0, & 1, & 0\end{array}\right)=\left(\mathbf{5 6}_{\mathbf{B}} \oplus \mathbf{5 6}_{\mathbf{F}}\right)$ of $\mathrm{SU}(5 / 2)$ contains ( 0000,1$)$, which is typical, and ( 00001,0$)$, which is atypical irrep of $\mathrm{SU}(5 / 1)$ simultaneously, such as

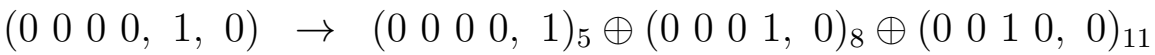

$$
\begin{aligned}
& \oplus\left(\begin{array}{lllll}
0 & 1 & 0 & 0, & 0
\end{array}\right)_{14} \oplus\left(\begin{array}{lllll}
1 & 0 & 0 & 0 & 0
\end{array}\right)_{17} \oplus\left(\begin{array}{lllll}
0 & 0 & 0 & 0, & 0
\end{array}\right)_{20} \text {. }
\end{aligned}
$$

As gauge bosons are identified with the adjoint rep (l $\left.\begin{array}{lll}0 & 0 & 1\end{array}\right)$ of SU(5) model, we introduce the adjoint rep $\left(\begin{array}{llll}1 & 0 & 0 & 0,-1\end{array}\right)$ of $\mathrm{SU}(5 / 1)[18]$. The branching rule is

\begin{tabular}{|c|c|c|c|}
\hline & $\mathrm{SU}(5 / 1)$ & $\mathrm{SU}(5) \otimes \mathrm{U}(1)$ & $\mathrm{SU}_{c}(3) \otimes \mathrm{SU}_{w}(2) \otimes \mathrm{U}_{Y}(1)$ \\
\hline gnd $>$ & 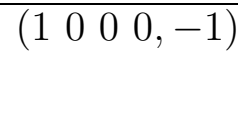 & $\left(\begin{array}{llll}1 & 0 & 0 & 0\end{array}\right)_{-1}$ & $\begin{array}{l}\left(\begin{array}{ll}1 & 0\end{array}\right)(0)_{1 / 3} \\
\left(\begin{array}{ll}0 & 0\end{array}\right)(1)_{-1 / 2}\end{array}$ \\
\hline $1 \mathrm{st}>$ & $\left(\begin{array}{llll}1 & 0 & 0 & 1,-1\end{array}\right)$ & $\left(\begin{array}{llll}1 & 0 & 0 & 1\end{array}\right)_{0}$ & 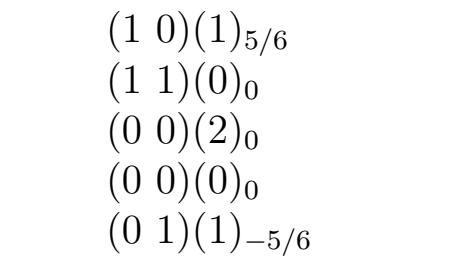 \\
\hline & $\left(\begin{array}{lllll}0 & 0 & 0 & 0, & 0\end{array}\right)$ & $\left(\begin{array}{llll}0 & 0 & 0 & 0\end{array}\right)_{0}$ & $\left(\begin{array}{ll}0 & 0\end{array}\right)(0)_{0}$ \\
\hline $2 \mathrm{nd}>$ & $\left(\begin{array}{lllll}0 & 0 & 0 & 1, & 0\end{array}\right)$ & $\left(\begin{array}{llll}0 & 0 & 0 & 1\end{array}\right)_{1}$ & 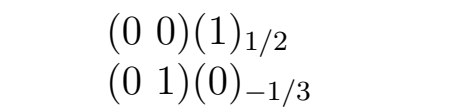 \\
\hline
\end{tabular}

Indeed, the first floor of Eq. (17) contains the adjoint rep $\left(\begin{array}{llll}1 & 0 & 0 & 1\end{array}\right)=\mathbf{2 4}$ of $\mathrm{SU}(5)$ which represents the gauge bosons.

\section{$4 \quad \mathrm{SU}(5 / 3)$ Unification}

$\mathrm{SU}(5 / 3)$ has fifteen positive/negative odd roots such that an typical irrep of the superalgebra has sixteen floors. The lowest dimensional typical irrep of $\mathrm{SU}(5 / 3)$ is 


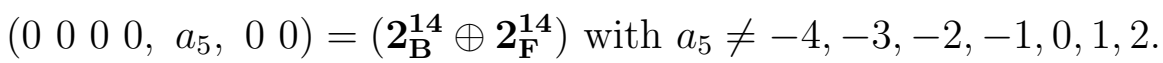

Since the $\mathrm{SU}(5 / 3)$ superalgebra has $\mathrm{SU}(5 / 1) \otimes \mathrm{SU}(2)$ as a subalgebra, one may expect to find one irrep of $\mathrm{SU}(5 / 3)$ which can accommodate three generations of fundamental particles. In particular we will concentrate on the $\mathrm{SU}(5 / 1) \otimes \mathrm{SU}(2)$ branching from SU(5/3) because both the irreps $\left(\begin{array}{llll}0 & 0 & 0 & 0,1\end{array}\right)$ and $\left(\begin{array}{llll}0 & 0 & 0 & 1,0\end{array}\right)$ of $\mathrm{SU}(5 / 1)$ superalgebra can fit $\left[5^{*}+\mathbf{1 0}\right]$ reps of SU(5) GUT separately. As a natural extension of Eq. (16), we note on an atypical irrep $\left(\begin{array}{llllll}0 & 0 & 0 & 0,1, & 0 & 0\end{array}\right)=\left(\mathbf{1 3 6}_{\mathbf{B}} \oplus \mathbf{1 3 6}_{\mathbf{F}}\right)$ of $\mathrm{SU}(5 / 3)$ which has the explicit weight system as follows

$$
\begin{aligned}
& \mid \text { gnd }>\quad\left(\begin{array}{lllllll}
0 & 0 & 0 & 0,1, & 0 & 0
\end{array}\right) \\
& \text { | 1st > (0 } 0001,1,10) \\
& \text { | 2nd > (0 } 0010,2,200)
\end{aligned}
$$

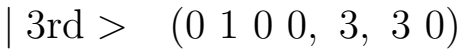

$$
\begin{aligned}
& \text { | 4th > (1 } 0000,4,40) \\
& \text { | } 5 \text { th }>\quad(0000,5,50) \text {. }
\end{aligned}
$$

The branching rule $\mathrm{SU}(5 / 3) \rightarrow \mathrm{SU}(5 / 1) \otimes \mathrm{SU}(2)$ of Eq. (18) is given by

$$
\begin{aligned}
& \left(\begin{array}{llllll}
0 & 0 & 0 & 0,1,1,0 & 0
\end{array}\right) \rightarrow\left(\begin{array}{llll}
0 & 0 & 0 & 0,1
\end{array}\right)(0) \oplus\left(\begin{array}{llll}
0 & 0 & 0 & 1,0
\end{array}\right)(1) \oplus\left(\begin{array}{lllll}
0 & 0 & 1 & 0,0
\end{array}\right)(2) \\
& \oplus\left(\begin{array}{lllll}
0 & 1 & 0 & 0,0 & 0
\end{array}\right)(3) \oplus\left(\begin{array}{lllll}
1 & 0 & 0 & 0,0 & 0
\end{array}\right)(4) \oplus\left(\begin{array}{lllll}
0 & 0 & 0 & 0,0 & 0
\end{array}\right)(5) .
\end{aligned}
$$

Note that the irrep ( $\left.\begin{array}{llllll}0 & 0 & 0 & 0,1, & 0 & 0\end{array}\right)$ contain one typical irrep $\left(\begin{array}{llll}0 & 0 & 0 & 0,1\end{array}\right)\left(\begin{array}{l}0 \\ )\end{array}\right)$ and one atypical irrep $\left(\begin{array}{lllll}0 & 0 & 0 & 1, & 0\end{array}\right)(1)$ at $\mathrm{SU}(5 / 1) \otimes \mathrm{SU}(2)$ stage. If we break the $\mathrm{SU}(2)$ flavor part, then it can be easily shown that the irrep $\left(\begin{array}{llllll}0 & 0 & 0 & 0,1,00\end{array}\right)$ contains three copies of $\left(5^{*} \oplus \mathbf{1 0}\right)$ reps of $\mathrm{SU}(5) \mathrm{GUT}$.

On the other hand, the branching rule $\mathrm{SU}(5 / 3) \rightarrow \mathrm{SU}(5 / 1) \otimes \mathrm{SU}(2)$ for the adjoint rep $\left(\begin{array}{llllll}1 & 0 & 0 & 0,0,0 & 1\end{array}\right)$ is given by

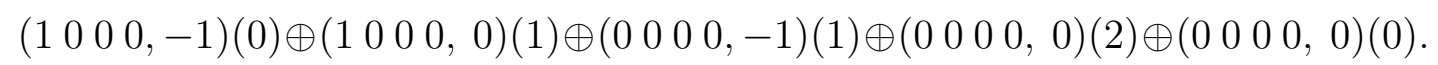


Similar to the SU(5/1) case in Eq. (17), the first floor of $\left(\begin{array}{lllll}1 & 0 & 0 & 0,-1\end{array}\right)(0)$ in Eq. (20) also contains the adjoint rep $\left(\begin{array}{llll}1 & 0 & 0 & 1\end{array}\right)=\mathbf{2 4}$ of $\mathrm{SU}(5)$.

\section{Conclusion}

In this paper we have studied a group theoretical framework for unified model building including three generations of fundamental particles in SU(5/3). Elementary particles is not our field of specialization, so we leave it to the specialists to consider this representation theoretic picture as a basis for real models. It is only after such considerations that the proposed $\mathrm{SU}(5 / 3)$ superalgebra, which has $\mathrm{SU}(5 / 1) \otimes \mathrm{SU}(2)$ substructure, can be regarded as an interesting part of physics, or whether it is just a mathematical coincidence. In fact the $\mathrm{SU}(5 / 3)$ superalgebra could describe supersymmetric $\mathrm{SU}(5 / 1)$ unification, which includes the well-known SU(5) model [3], while the SU(2) flavor part accommodates all known three generations.

In conclusion, we have studied the lowest dimensional typical irrep and its atypical irreps of $\mathrm{SU}(5 / 3)$ superalgebra as a possible unified gauge theory that has a natural $\mathrm{SU}(5 / 1) \otimes \mathrm{SU}(2)$ subalgebra structure. As a result, we have explicitly shown that all known three generations of fundamental particles could be in just one irrep

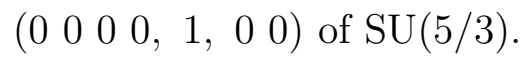

\section{Acknowledgments}

Y.-J. Park was supported by the National Research Foundation of Korea (NRF) grant funded by the Korea government (MEST) through the Center for Quantum Spacetime (CQUeST) of Sogang University with grant number 2005-0049409. 


\section{References}

[1] S.L. Glashow, Nucl. Phys. 22, 519 (1961); A. Salam and J.C. Ward, Phys. Lett. 13, 168 (1964); S. Weinberg, Phys. Rev. Lett. 19, 1264 (1967); A. Salam, in Elementary Particle Physics, N. Svartholm, ed. (Nobel Symposium No. 8, Almquist and Wiksell, Stockholm, 1968), p. 367.

[2] S. Weinberg, Phys. Rev. Lett. 31, 494 (1973); D. J. Gross and F. Wilczek, Phys. Rev. D8, 3633 (1973); Phys. Rev. Lett. 30, 1343 (1973); H. Fritzsch, M. Gell-Mann and H. Leutwyler, Phys. Lett. B47, 365 (1973).

[3] H. Georgi and S. L. Glashow, Phys. Rev. Lett. 32, 438 (1974).

[4] H. Fritzsch and P. Minkowski, Ann. Phys. 93, 193 (1975); H. Georgi, Particles and Fields (APS/DPF Williamsburg), ed. C. E. Carlson (AIP, New York, 1975).

[5] F. Gusey, P. Ramond, and P. Sikivie, Phys. Lett. B60, 177 (1975).

[6] G.G. Ross, Grand Unified Theories (Benjamin Cummings Publishing, California, 1985).

[7] V. Kac, Adv. in Math. 26, 8 (1977); Commun. Math. Phys. 53, 31 (1977).

[8] Y.A. Gol'fand and E.P. Likhtman, Pis'ma Zh. Eksp. Theor. Fiz. 13, 452(1971)[JETP Lett. 13, 323(1971)]; A. Neveu and J. H. Schwarz, Nucl. Phys. B31, 86 (1971); P. Ramond, Phys. Rev. D3, 2415 (1971); P. G. O. Freund and I. Kaplansky, J. Math. Phys. 17, 228 (1976); S. Deser and B. Zumino, Phys. Lett. 62B, 335 (1976); D. Z. Freedman, P. van Nieuwenhuizen, and S. Ferrara, Phys. Rev. D13, 3214 (1976); Y. Ne'eman, Phys. Lett. 81B, 190 (1979); F. Iachello, Phys. Rev. Lett. 44, 772 (1980); A.B. Balantekin, I. Bars, and F. Iachello, ibid. 47, 19 (1981); P. van Nieuwenhuizen, Phys. Rep. 68, 189 (1981); M. B. Green and J. H. Schwarz, Nucl. Phys. B198, 474 (1982); Y. Ne'eman, S. Sternberg, and D. Fairlie, Phys. Rep. 406, 303 (2005); J. Thierry-Mieg, PTA/07-058 (2008); R. Kitano, M. Luty and Y. Nakai, JHEP 08, 111 (2012). 
[9] J. P. Hurni and B. Morel, J. Math. Phys. 24, 157 (1983); C. H. Kim, K. Y. Kim, W. S. l'Yi, Y. Kim, and Y. J. Park, J. Math. Phys. 27, 2009 (1986).

[10] J. Strathdee, Int. J. Mod. Phys. A2, 173 (1987).

[11] E. Cremmer, in Superspace and Supergravity, edited by S. Hawking and M. Rocek, p.267 (Cambridge University Press, London, England, 1980); C. H. Kim, K. Y. Kim, W. S. l'Yi, Y. Kim, and Y. J. Park, Mod. Phys. Lett. A3, 1005 (1988); C. H. Kim, K. Y. Kim, Y. Kim, and Y. J. Park, Phys. Rev. D39, 2967 (1989); C. H. Kim, K. Y. Kim, Y. Kim, H. W. Lee, W. S. l'Yi, and Y. J. Park, Phys. Rev. D40, 1969 (1989); C. H. Kim, Y. J. Park, K. Y. Kim, Y. Kim, and W. S. l'Yi, Phys. Rev. D44, 3169 (1991); C. H. Kim, Y. J. Park, and Y. Kim, Mod. Phys. Lett. A10, 1929 (1995); C. H. Kim and Y. J. Park, Mod. Phys. Lett. A12, 851 (1997).

[12] J. Schwarz, Phys. Lett. B367, 97 (1996); E. Witten, Nucl. Phys. B460, 335 (1995); C. Vafa, Nucl. Phys. B469, 403 (1996); J. Polchiski, "Tasi Lectures on D-branes", hep-th/9611050. H.J. Boonstra, B. Peeters, and K. Skenderis, Nucl. Phys. B533, 127 (1998) J. Maldacena, Adv. Theor. Math. Phys. 2, 231 (1998).

[13] I. Bars, Phys. Rev. D54, 5203 (1996).

[14] V. Kac, Adv. in Math. 139, 1 (1998); V. Kac and A. Rudakov, Comm. Math. Phys. 222, 611 (2001); V. Kac, ICM 2002 1, 319 (2002); V. Kac and A. Rudakov, Tran. Groups, 7, 67 (2002), T. Larsson, math-ph/0202023 (2002); V. Kac, in Visions in Mathematics, p162 (Modern Birkhauser Classics, 2010)

[15] P.D. Javis, arXiv:1109.4881 (2011); J. Phys. A: Math. Theor. 45, 322001 (2012).

[16] P.H. Dondi and P.D. Jarvis, Z. Physik C4, 201 (1980).

[17] N.A. Batakis, A.A. Kehagias and G. Zoupanos, Phys. Lett. B315, 319 (1993).

[18] N.I. Stoilova and J. Van der Jeugt, Int. J. Theo. Phys. 44, 1157 (2005).

[19] J. Thierry-Mieg, Phys. Lett. 138B, 393 (1984). 